\title{
A NONLOCAL TIMOSHENKO BEAM THEORY FOR VIBRATION ANALYSIS OF THICK NANOBEAMS USING DIFFERENTIAL TRANSFORM METHOD
}

\author{
Farzad Ebrahimi, Parisa Nasirzadeh \\ Department of Mechanical Engineering, Faculty of Engineering, Imam Khomeini International University, Qazvin, Iran \\ e-mail: febrahimy@eng.ikiu.ac.ir
}

\begin{abstract}
This article presents the solution for free vibration of nanobeams based on Eringen nonlocal elasticity theory and Timoshenko beam theory. The small scale effect is considered in the first theory, and the transverse shear deformation effects as well as rotary inertia are taken into account in the latter one. Through variational formulation and the Hamilton principle, the governing differential equations of free vibration of the nonlocal Timoshenko beam and the boundary conditions are derived. The obtained equations are solved by the differential transformation method (DTM) for various frequency modes of the beams with different end conditions. In addition, the effects of slenderness and on vibration behavior are presented. It is revealed that the slenderness affects the vibration characteristics slightly whilst the small scale plays a significant role in the vibration behavior of the nanobeam.
\end{abstract}

Keywords: free vibration, nanobeam, Eringen nonlocal elasticity theory

\section{Introduction}

Nanostructures have significant mechanical, electrical and thermal performances that are superior to conventional structural materials. They have attracted much attention in modern science and technology. For example, in micro/nano electromechanical systems (MEMS/NEMS); nanostructures have been used in many areas including communications, machinery, information technology, biotechnology technologies, etc. So far, three main methods have been provided to study the mechanical behavior of nanostructures. These are the atomistic (Baughman et al., 2002), semi-continuum ( $\mathrm{Li}$ and Chou, 2003) and continuum models (Wang and Cai, 2006). However, both atomistic and semi-continuum models are computationally expensive and are not suitable for analyzing large scale systems.

Due to the inherent size effects, at nanoscale, the mechanical characteristics of nanostructures are often significantly different from their behavior at macroscopic scale. Such effects are essential for nanoscale materials or structures and the influence on nano-instruments is great (Maranganti and Sharma, 2007). Generally, theoretical studies on size effects at nanoscale are by means of surface effects (Zhu et al., 2009), strain gradients in elasticity (Mindlin, 1964) and plasticity (Aifantis, 1984) as well as nonlocal stress field theory (Eringen, 1983, 1972a), etc. Unfortunately, the classical continuum theories are deemed to fail for these nanostructures, because length dimensions at nano scale are often sufficiently small such that call the applicability of classical continuum theories into the question. Consequently, the classical continuum models need to be extended to consider the nanoscale effects. This can be achieved through the nonlocal elasticity theory proposed by Eringen (1972a) which considers the size-dependent effect. According to this theory, the stress state at a reference point is considered as a function of strain states of all points in the body. This nonlocal theory is proved to be in accordance with the atomic model of lattice dynamics and with experimental observations on phonon dispersion (Eringen, 1983). In nonlocal theory, nonlocal nanoscale in the constitutive equation could be considered simply 
as a material-dependent parameter. The ratio of internal characteristic scale (such as the lattice parameter, C-C bond length, granular distance, etc.) to external characteristic scale (such as crack length, wave length, etc.) is defined within a nonlocal nanoscale parameter. If the internal characteristic scale is much smaller than the external characteristic scale, the nonlocal nanoscale parameter approaches zero and the classical continuum theory is recovered.

For analyzing these nanoscale beams, Euler-Bernoulli and Timoshenko beam theories appear to be inadequate, since they are scale free. For this problem, continuum mechanics is needed, and one of the efficient theories for nonlocal continuum mechanics is Eringen's (Eringen, 1983; 1972a,b) theory which allows small scale effect by indicating that stress at one point is a function of strain at all points of the body.

In the recent years, nanobeams and carbon nanotubes (CNTs) have held a wide variety of potential applications (Zhang et al., 2004; Wang, 2005; Wang and Varadan, 2006) such as sensors, actuators, transistors, probes, and resonators in nanoelectromechnical systems (NEMSs). Thus, establishing an accurate model of nanobeams is a key issue for successful NEMS design. As a result, nanotechnological research on free vibration properties of nanobeams is important because such components can be used as design components in nano-sensors and nano-actuators. Furthermore, many researchers worked on bending, buckling and vibration of beam-like elements (Peddieson et al., 2003; Liew et al., 2008; Xu, 2006; Amara et al., 2010) and in some papers the Euler-Bernoulli theory has been applied for vibration of nanobeams (Lu et al., 2006; Zhang et al., 2005; Xu, 2006). But they used the Euler-Bernoulli theory which does not account transverse shear force and rotary inertia which are significant in stubby beams and high vibration frequencies. So in this paper, we used Timoshenko beam theory and the governing equations and boundary conditions for free vibration of a nonlocal Timoshenko beam have been derived via Hamilton's principle. To the author's best knowledge, there is no work reported on the application of DTM on vibration analysis of nonlocal Timoshenko beams with various boundary conditions. Furthermore, the solution procedure in this study is the differential transformation method (DTM) which is a semi analytical-numerical technique depending on Taylor series expansion. This method was first introduced by Zhou (1986) in his study about electrical circuits, and this method has the advantage of its simplicity in use as well as high accuracy. The results in this paper are provided by a MATLAB code with respect to DTM rules, for the first time.

\section{Nonlocal Timoshenko beam equations and boundary conditions}

Consider a beam with length $L$ and cross sectional area of $A$. Based on Timoshenko beam theory, strain-displacement and strain energy relations are as follows (Wang et al., 2000)

$$
\varepsilon_{x x}=z \frac{d \phi}{d x} \quad \gamma_{x z}=\phi+\frac{d w}{d x}
$$

in which $x$ is the longitudinal coordinate measured from the left end of the beam and $z$ is the coordinate measured from the mid-plane of the beam, $w$ represents the transverse displacement and $\phi$ is rotation of the beam due to bending, $\varepsilon_{x x}$ is the normal strain, $\gamma_{x z}$ is the transverse shear strain, $\sigma_{x x}$ is normal stress and $\sigma_{x z}$-transverse shear stress. The strain energy relation is as follows (Leissa and Qatu, 2011)

$$
U=\frac{1}{2} \int_{0}^{L} \int_{A}\left(\sigma_{x x} \varepsilon_{x x}+\sigma_{x z} \gamma_{x z}\right) d A d x
$$


After substituting equations (2.1) into equation (2.2) and putting the bending moment and shear force into relation (2.2), the strain energy becomes

$$
\begin{aligned}
& U=\frac{1}{2} \int_{0}^{L} \int_{A}\left(\sigma_{x x} z \frac{d \phi}{d x}+\sigma_{x z}\left(\phi+\frac{d w}{d x}\right)\right] d A d x=\int_{0}^{L} \frac{1}{2}\left[M \frac{d \phi}{d x}+Q\left(\phi+\frac{d w}{d x}\right)\right] d x \\
& M=\int_{A} \sigma_{x x} z d A \quad Q=\int_{A} \sigma_{x z} d A
\end{aligned}
$$

where $M$ is the bending moment and $Q$ is the shear force. The kinetic energy $T$, by assuming free harmonic motion and rotary inertia effect, is

$$
T=\frac{1}{2} \int_{0}^{L}\left(\rho A \omega^{2} w^{2}+\rho I \omega^{2} \phi^{2}\right) d x
$$

in which $\omega$ is the circular frequency of vibration and $\rho$ and $I$ are the mass density and the second moment of area of the beam, respectively. Applying Hamilton's principle (Chow, 2013), requires

$$
\delta(T-U)=0=\int_{0}^{L}\left[-M \frac{d \delta \phi}{d x}-Q\left(\delta \phi+\frac{d \delta w}{d x}\right)+\rho A \omega^{2} w \delta w+\rho I \omega^{2} \phi \delta \phi\right] d x
$$

After performing integration by parts, we reach

$$
\left.0=\int_{0}^{L}\left[\left(\frac{d M}{d x}-Q+\rho I \omega^{2} \phi\right) \delta \phi+\frac{d Q}{d x}+\rho A \omega^{2} w\right) \delta w\right] d x-[M \delta \phi]_{0}^{L}-[Q \delta w]_{0}^{L}
$$

This results in the following equations

$$
\frac{d M}{d x}=Q-\rho I \omega^{2} \phi \quad \frac{d Q}{d x}=-\rho A \omega^{2} w
$$

And the boundary conditions are in two forms of below relations

$$
\begin{array}{llll}
\text { Either } & w=0 & \text { or } & Q=0 \\
\text { Either } & \phi=0 & \text { or } & M=0
\end{array}
$$

As can be seen, the equations appear to be the same as in local Timoshenko beam theory, but the shear force and bending moment expressions in nonlocal beam theory must be different. The constitutive equation of classical elasticity is an algebraic relationship between stress and strain tensors while Eringen nonlocal elasticity includes spatial integrals which indicate the average effect of strain of all points of the body to the stress tensor at the given point (Eringen, 1972b; 1983). Since the spatial integrals in constitutive equations are mathematically difficult to solve, they can be converted into equal differential constitutive equations under certain conditions. The nonlocal constitutive stress-strain relation for an elastic material in the one dimensional case beam can be simplified as (Eringen, 1983)

$$
\sigma_{x x}-\left(e_{0} a\right)^{2} \frac{d^{2} \sigma_{x x}}{d x^{2}}=E \varepsilon_{x x}
$$

in which $E$ is the Young modulus, $e_{0} a$ is the scale coefficient that incorporates the small scale effect, $a$ represents the internal characteristic length and $e_{0}$ is a constant appropriate to each material which is measured experimentally. The local and nonlocal constitutive shear strain- 
-stress relations are the same, since form of the Eringen nonlocal constitutive model cannot be applied in the $z$ direction

$$
\sigma_{x z}=G \gamma_{x z}
$$

in which $G$ is the shear modulus. After multiplying the term $(z d A)$ and integrating over the area $A$, equation (2.9) becomes

$$
M-\left(e_{0} a\right)^{2} \frac{d^{2} M}{d x^{2}}=E I \frac{d \phi}{d x}
$$

By integrating equation (2.10) over the area, we obtain

$$
Q=K_{s} G A\left(\phi+\frac{d w}{d x}\right)
$$

in which $K_{s}$ is the shear correction factor in the Timoshenko beam theory in order to compensate for the error in assuming equal shear stress or strain in the whole beam thickness. Now by substituting equations (2.7) into equation (2.11), the moment can be reached as below

$$
M=E I \frac{d \phi}{d x}-\left(e_{0} a\right)^{2}\left(\rho A \omega^{2} w+\rho I \omega^{2} \frac{d \phi}{d x}\right)
$$

And by utilizing equations (2.12) and (2.13) in Timoshenko beam equations (2.7), the governing equation for the vibration of nonlocal Timoshenko beam may be obtained as below

$$
\begin{aligned}
& E I \frac{d^{2} \phi}{d x^{2}}-K_{s} G A\left(\phi+\frac{d w}{d x}\right)+\rho I \omega^{2} \phi-\left(e_{0} a\right)^{2}\left(\rho A \omega^{2} \frac{d w}{d x}+\rho I \omega^{2} \frac{d^{2} \phi}{d x^{2}}\right)=0 \\
& K_{s} G A\left(\frac{d \phi}{d x}+\frac{d^{2} w}{d x^{2}}\right)+\rho A w \omega^{2}=0
\end{aligned}
$$

On the basis of equation (2.8) and due to various endings of the beam, e.g. for a simply supported end, we have

$$
w=0 \quad M=E I \frac{d \phi}{d x}-\left(e_{0} a\right)^{2}\left(\rho A \omega^{2} w+\rho I \omega^{2} \frac{d \phi}{d x}\right)=0
$$

and for a clamped end

$$
w=0 \quad \phi=0
$$

and for a free end

$$
\begin{aligned}
& M=E I \frac{d \phi}{d x}-\left(e_{0} a\right)^{2}\left(\rho A \omega^{2} w+\rho I \omega^{2} \frac{d \phi}{d x}\right)=0 \\
& Q=K_{s} G A\left(\phi+\frac{d w}{d x}\right)=0
\end{aligned}
$$

\section{Non-dimensional parameters}

The non-dimensional parameters contributes to simplification of the equations and to the making of comparisons in the studies possible. The non-dimensional parameters are introduced as the following terms

$$
\begin{array}{lll}
\bar{x}=\frac{x}{L} & \bar{w}=\frac{w}{L} & \lambda^{2}=\omega^{2} \frac{\rho A L^{4}}{E I} \\
\Omega=\frac{E I}{K_{s} G A L^{2}} & \alpha=\frac{e_{0} a}{L} & \varepsilon=\frac{L \sqrt{A}}{\sqrt{I}}
\end{array}
$$


where $\lambda^{2}$ is frequency parameter, $\Omega$ - shear deformation parameter, $\alpha$ - scaling effect arameter, $\varepsilon$ - slenderness ratio.

By applying the non-dimensional parameters to governing equations (2.14), the following relations are obtained

$$
\begin{aligned}
& \Omega\left(1-\frac{\alpha^{2} \lambda^{2}}{\varepsilon^{2}}\right) \frac{d^{2} \phi}{d \bar{x}^{2}}+\left(\frac{\Omega \lambda^{2}}{\varepsilon^{2}}-1\right) \phi-\left(\alpha^{2} \lambda^{2} \Omega+1\right) \frac{d \bar{w}}{d \bar{x}}=0 \\
& \frac{d \phi}{d \bar{x}}+\frac{d^{2} \bar{w}}{d \bar{x}^{2}}+\lambda^{2} \Omega \bar{w}=0
\end{aligned}
$$

Also boundary conditions equations (2.15)- (2.17) appear for the simply supported end as

$$
\bar{w}=0 \quad M=\left(\Omega-\frac{\Omega \alpha^{2} \lambda^{2}}{\varepsilon^{2}}\right) \frac{d \phi}{d \bar{x}}-\Omega \alpha^{2} \lambda^{2} \bar{w}=0
$$

And for the clamped end as

$$
\bar{w}=0 \quad \phi=0
$$

And for the free end as

$$
M=\left(\Omega-\frac{\Omega \alpha^{2} \lambda^{2}}{\varepsilon^{2}}\right) \frac{d \phi}{d \bar{x}}-\Omega \alpha^{2} \lambda^{2} \bar{w}=0 \quad Q=K_{s} G A\left(\phi+\frac{d \bar{w}}{d \bar{x}}=0\right.
$$

\section{Differential transformation method}

The differential transformation method is one of the useful techniques to solve differential equations with small calculation errors and capable of solving nonlinear equations with boundary condition value problems. Abdel-Halim Hassan (2002) applied the DTM to eigenvalues and normalized eigenfunctions. Also Wang (2013) and Chen and Ju (2004) used the method in their studies. The DTM is a transformation technique based on the Taylor series expansion and is a useful tool to obtain analytical solutions to differential equations. The DTM is proved to be a good computational tool for various engineering problems. Using the differential transformation technique, ordinary and partial differential equations can be transformed into algebraic equations from which a closed-form series solution can be obtained easily. In this method, certain

\begin{tabular}{|c|c|}
\hline Original function & Transformed function \\
\hline$f(x)=g(x \pm h(x)$ & $F(K)=G(K) \pm H(K)$ \\
\hline$f(x)=\lambda g(x)$ & $F(K)=\lambda G(K)$ \\
\hline$f(x)=g(x) h(x)$ & $F(K)=\sum^{K} G(K-l) H(l)$ \\
\hline$f(x)=\frac{d^{n} g(x)}{d n}$ & $F(K)=\frac{(k+n) !}{u} G(K+n)$ \\
\hline$f(x)=x^{n}$ & $F(K)=\delta(k-n)=\left\{\begin{array}{lll}0 & \text { if } & k \neq n \\
1 & \text { if } & k=n\end{array}\right.$ \\
\hline
\end{tabular}
transformation rules are applied to both the governing differential equations of motion and the boundary conditions of the system in order to transform them into a set of algebraic equations as presented in Table 1 and 2 .

Table 1. Basics of the differential transform method (Chen and Ju, 2004) 
Table 2. Transformed boundary conditions (BC) based on DTM (Chen and Ju, 2004)

\begin{tabular}{|c|c|c|c|}
\hline \multicolumn{2}{|c|}{$X=0$} & \multicolumn{2}{c|}{$X=1$} \\
\hline $\begin{array}{c}\text { Original } \\
\text { BC }\end{array}$ & $\begin{array}{c}\text { Transformed } \\
\text { BC }\end{array}$ & $\begin{array}{c}\text { Original } \\
\text { BC }\end{array}$ & $\begin{array}{c}\text { Transformed } \\
\text { BC }\end{array}$ \\
\hline \hline$f(0)=0$ & $F[0]=0$ & $f(1)=0$ & $\sum_{k=0}^{\infty} F[k]=0$ \\
$\frac{d f}{d x}(0)=0$ & $F[1]=0$ & $\frac{d f}{d x}(1)=0$ & $\sum_{k=0}^{\infty} k F[k]=0$ \\
$\frac{d^{2} f}{d x^{2}}(0)=0$ & $F[2]=0$ & $\frac{d^{2} f}{d x^{2}}(1)=0$ & $\sum_{k=0}^{\infty} k(k-1) F[k]=0$ \\
$\frac{d^{3} f}{d x^{3}}(0)=0$ & $F[3]=0$ & $\frac{d^{3} f}{d x^{3}}(1)=0$ & $\sum_{k=0}^{\infty} k(k-1)(k-2) F[k]=0$ \\
\hline
\end{tabular}

The solution of these algebraic equations gives the desired results of the problem. It is different from the high-order Taylor series method because the Taylor series method requires symbolic computation of necessary derivatives of data functions and is expensive for large orders.

The basic definitions and the application procedure of this method can be introduced as follows:

The transformation equation of the function $f(x)$ can be defined as (Chen and Ju, 2004)

$$
F[k]=\left.\frac{1}{k !} \frac{d^{k} f(x)}{d x^{k}}\right|_{x=x_{0}}
$$

where $f(x)$ the original function and $F[k]$ is the transformed function.

The inverse transformation is defined as

$$
f(x)=\sum_{k=0}^{\infty}\left(x-x_{0}\right)^{k} F[k]
$$

Combining equations (4.1) and (4.2), one obtains

$$
f(x)=\left.\sum_{k=0}^{\infty} \frac{\left(x-x_{0}\right)^{k}}{k !} \frac{d^{k} f(x)}{d x^{k}}\right|_{x=x_{0}}
$$

Considering equation (4.3), it is noticed that the concept of the differential transform is derived from Taylor series expansion. In actual application, the function $f(x)$ is expressed by a finite series, and equation (4.3) can be written as follows

$$
f(x)=\left.\sum_{k=0}^{n} \frac{\left(x-x_{0}\right)^{k}}{k !} \frac{d^{k} f(x)}{d x^{k}}\right|_{x=x_{0}}
$$

which implies that the term in relation (4.5) is negligible

$$
f(x)=\left.\sum_{k=n+1}^{\infty} \frac{\left(x-x_{0}\right)^{k}}{k !} \frac{d^{k} f(x)}{d x^{k}}\right|_{x=x_{0}}
$$

In this study, the natural frequencies determine the value of $n$. 


\section{Solution with DTM}

According to the DTM rules given in Table 1, equations (3.1) will be transformed into the following equations

$$
\begin{aligned}
& \Omega\left(1-\frac{\alpha^{2} \lambda^{2}}{\varepsilon^{2}}\right)(k+1)(k+2) \Phi(k+2)+\left(\frac{\Omega \lambda^{2}}{\varepsilon^{2}}-1\right) \Phi(k)-\left(\alpha^{2} \lambda^{2} \Omega+1\right)(k+1) \bar{W}(k+1)=0 \\
& (K+1) \Phi(k+1)+(k+1)(k+2) \bar{W}(k+2)+\lambda^{2} \Omega \bar{W}(k)=0
\end{aligned}
$$

The rules of the DTM for defining boundary conditions are given in Table $2 . \bar{W}(k)$ and $\Phi(k)$ are transforms of $w(x)$ and $\phi(x)$, respectively. By substituting values for $k=0,1,2, \ldots, \alpha=0$, $\varepsilon=34.641$ and $\Omega=0.2436$ into equations (5.1), we can evaluate the amounts of $\bar{W}(2), \bar{W}(3), \ldots$ and $\Phi(2), \Phi(3), \ldots$ in terms of $\omega^{2}$ and some constants like $c_{1}, \ldots$ The values can be achieved with a computer program, and after substituting $\bar{W}(i)$ and $\Phi(i)$ into boundary conditions the following equation is obtained

$$
N_{r} 1^{(n)}(\omega) c_{1}+N_{r} 2^{(n)}(\omega) c_{2}=0 \quad r=1,2, \ldots, n
$$

in which $N_{s}$ are polynomials in terms of $\omega$ corresponding to the $n$-th term. When solving equation (5.2) in matrix form, the following eigenvalue equation may be obtained

$$
\left|\begin{array}{ll}
N_{11}^{n}(\omega) & N_{12}^{n}(\omega) \\
N_{21}^{n}(\omega) & N_{22}^{n}(\omega)
\end{array}\right|=0
$$

The solution to equation (5.3) gives $\omega_{r}^{n}$ which is the $r$-th estimated eigenvalue for the $n$-th repeat. The number of repeats can be obtained by equation (5.4) as

$$
\left|\omega_{r}^{n}-\omega_{r}^{n-1}\right|<\delta
$$

In the present study, $\delta=0.0001$ and this shows the accuracy of calculations. With respect to the differential transformation method and the algorithm above, a MATLAB code has been developed in order to determine vibration characteristics of the nonlocal Timoshenko nanobeam.

\section{Results and discussion}

In the present study, the impact of the small scale coefficient as well as the effect of slenderness on the first, second and third frequencies of the nonlocal Timoshenko nanobeam are presented. Also, three types of boundary conditions are compared. In order to validate the computed results, a comparison between the present paper and the results obtained by Wang et al. (2007) is performed. The mechanical properties of the nonlocal Timoshenko nanobeam are given in Table 3.

Table 3. Mechanical properties of the nonlocal Timoshenko nanobeam (Wang et al., 2007)

\begin{tabular}{|c|c|c|}
\hline Property & Unit & \\
\hline \hline$E$ & $\mathrm{~T} \cdot \mathrm{Pa}$ & 5.5 \\
\hline$P$ & $\mathrm{~g} \cdot \mathrm{cm}^{-3}$ & 2.3 \\
\hline$\nu$ & - & 0.19 \\
\hline
\end{tabular}

Also, the Timoshenko shear correction factor $k_{s}$ is taken 0.563 . For calculating the exact difference between the results of the present paper and the available results in literature, relation (6.1) has been applied

$$
\% \text { difference }=100 \cdot \frac{\mid \text { reference }- \text { present } \mid}{\text { present }}
$$


As shown by the comparisons given in Tables 4 and 5, a close correlation between these results validates the proposed method of solution.

Table 4. First three nondimensional frequencies $\sqrt{\lambda}$ of the nonlocal Timoshenko beam for both clamped ends and $L / d=10$

\begin{tabular}{|c|c|c|c|c|c|c|c|c|c|}
\hline \multirow{3}{*}{$\alpha$} & \multicolumn{4}{|c|}{ Mode 1 } & \multicolumn{3}{c|}{ Mode 2 } & \multicolumn{3}{c|}{ Mode 3 } \\
\cline { 2 - 11 } & $\begin{array}{c}\text { pre- } \\
\text { sent }\end{array}$ & $\begin{array}{c}\text { Wang et al. } \\
(2007)\end{array}$ & $\begin{array}{c}\text { diff. } \\
{[\%]}\end{array}$ & $\begin{array}{c}\text { pre- } \\
\text { sent }\end{array}$ & $\begin{array}{c}\text { Wang et al. } \\
(2007)\end{array}$ & $\begin{array}{c}\text { diff. } \\
{[\%]}\end{array}$ & $\begin{array}{c}\text { pre- } \\
\text { sent }\end{array}$ & $\begin{array}{c}\text { Wang et al. } \\
(2007)\end{array}$ & $\begin{array}{c}\text { diff. } \\
{[\%]}\end{array}$ \\
\hline \hline 0 & 4.53 & 4.45 & 1.766 & 7.19 & 6.95 & 3.33 & 9.6 & 9.2 & 4.1 \\
\hline 0.1 & 4.4233 & 4.3471 & 1.72 & 6.67 & 6.4952 & 2.62 & 8.4 & 8.2 & 2.38 \\
\hline 0.3 & 3.83 & 3.7895 & 1.057 & 5 & 4.9428 & 1.14 & 5.95 & 5.846 & 1.74 \\
\hline 0.5 & 3.2657 & 3.242 & 0.725 & 4 & 3.994 & 0.15 & 4.75 & 4.6769 & 1.53 \\
\hline 0.7 & 2.85 & 2.8383 & 0.41 & 3.45 & 3.4192 & 0.89 & 4.05 & 3.9961 & 1.33 \\
\hline
\end{tabular}

Table 5. First nondimensional natural frequency $\sqrt{\lambda}$ of the nonlocal Timoshenko beam for two kinds of boundary conditions and $L / d=10$

\begin{tabular}{|c|c|c|c|c|c|c|}
\hline \multirow{2}{*}{$\alpha$} & \multicolumn{3}{|c|}{ Clamped-simple } & \multicolumn{3}{c|}{ Simple-free } \\
\cline { 2 - 7 } & $\begin{array}{c}\text { pre- } \\
\text { sent }\end{array}$ & $\begin{array}{c}\text { Wang et al. } \\
(2007)\end{array}$ & $\begin{array}{c}\text { diff. } \\
{[\%]}\end{array}$ & $\begin{array}{c}\text { pre- } \\
\text { sent }\end{array}$ & $\begin{array}{c}\text { Wang } \text { et al. } \\
(2007)\end{array}$ & $\begin{array}{c}\text { diff. } \\
{[\%]}\end{array}$ \\
\hline \hline 0 & 3.82 & 3.7845 & 0.929 & 3.08 & 3.0929 & 0.418 \\
\hline 0.1 & 3.73 & 3.6939 & 0.967 & 3.059 & 3.0243 & 1.13 \\
\hline 0.3 & 3.23 & 3.2115 & 0.5727 & 2.91 & 2.6538 & 8.8 \\
\hline 0.7 & 2.415 & 2.4059 & 0.37 & 2.4 & 2.0106 & 16 \\
\hline
\end{tabular}

In addition, the convergence of the differential transformation method is perused. In Fig. 1, the convergence of the third frequency of the nonlocal Timoshenko beam with both clamped ends is presented. It illustrates that the third frequency converges at the 46th repeat, while the first and the second frequencies converged before, in this example at the 29th and 37th repeats.

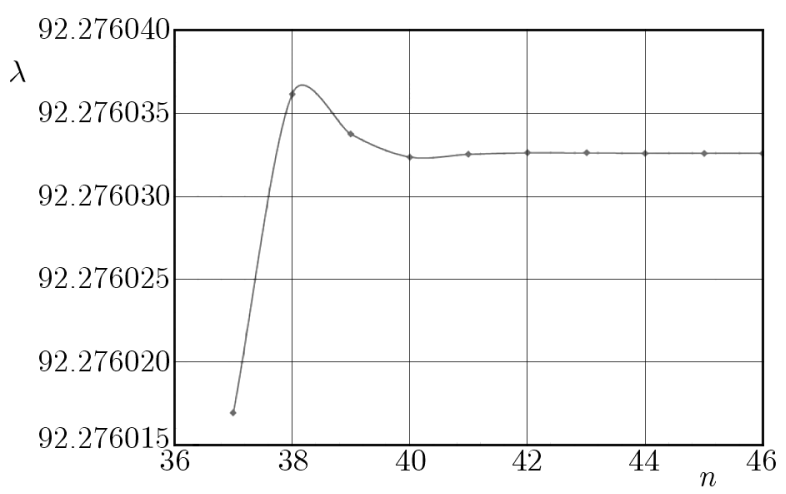

Fig. 1. Convergence of the third frequency, $L / d=10, \alpha=0$

The variables in governing equations (3.1) are $\alpha, \varepsilon$ and $\Omega . \alpha$ relates to the small scale effect, $\varepsilon$ is in terms of the slenderness $(L / d)$ and $\Omega$ relates to the mechanical properties and slenderness. So, it is possible to investigate the effects of slenderness and small scale on various frequencies and mode shapes of the nonlocal Timoshenko beam. Furthermore, determination of the magnitude of $e_{0}$ is significant due to its prominent effect on the small scale coefficient. Some researchers worked on estimating the magnitude of $e_{0} a$. For instance, Zhang et al. (2005) estimated the magnitude of the parameter for carbon nanotubes to be approximately 0.82 . In 
this study, we adopt $0 \leqslant \alpha<0.8$ in our investigations as reported by Lu et al. (2006). As Figs. 2a,b,c show when the coefficient $\alpha$ equals zero, the frequency of the nonlocal Timoshenko beam equals its local counterpart. As the coefficient increases, the frequency ratio decreases, which means that the nonlocal beam frequency becomes smaller than the local counterparts. This reduction is especially noticeable in higher modes and cannot be neglected. In sum, the small scale effect makes the beam more flexible since in nonlocal theory elastic springs link the atoms together (Liew et al., 2008).
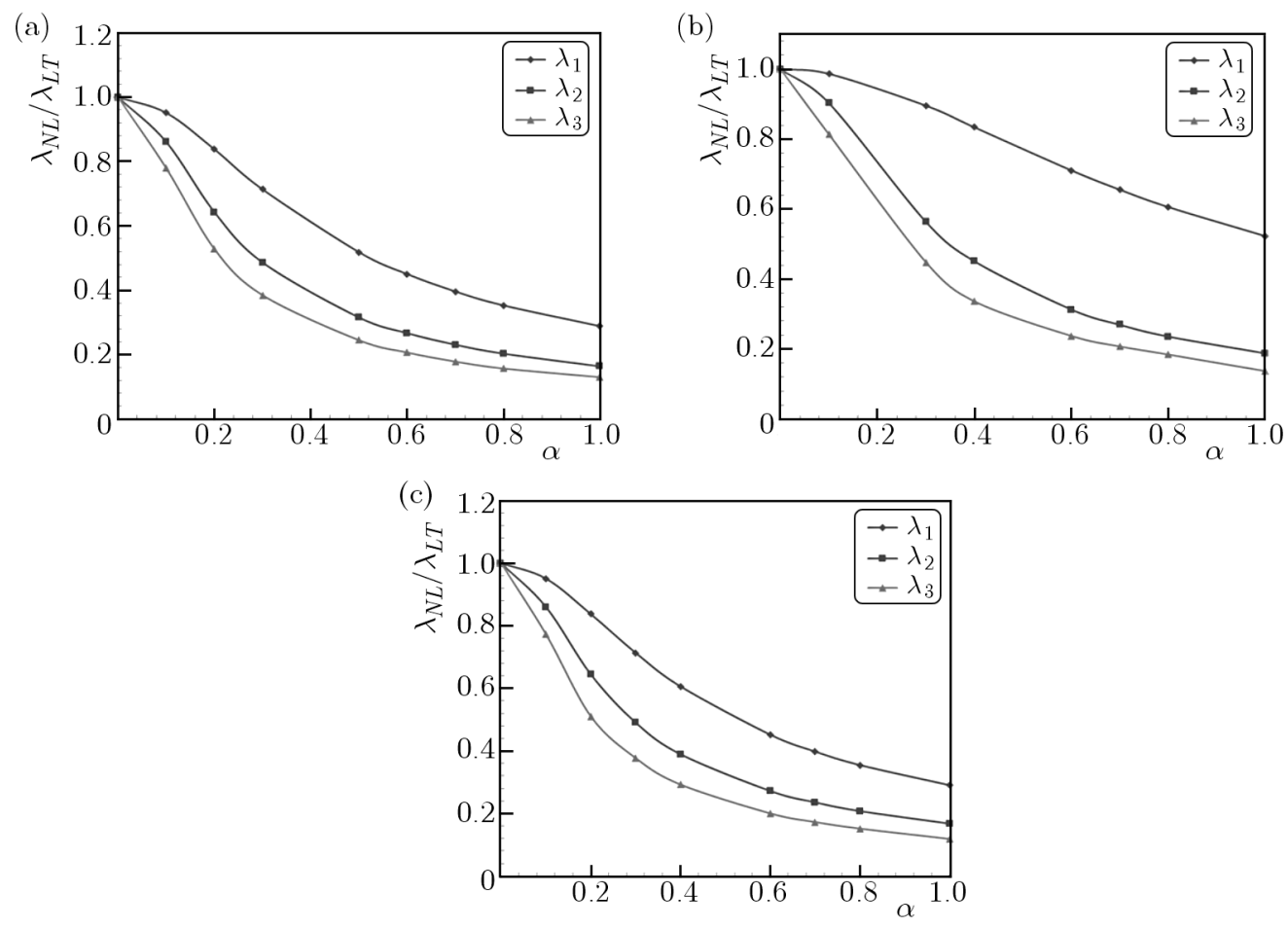

Fig. 2. Effect of small scale on different frequency modes, $L / d=10$ : (a) clamped ends, (b) simply supported beam, (c) clamped-simply beam

Figure 3 indicates that the small scale have significant effect on short beams and, as the beam gets longer, its impact becomes gradually negligible. So, the small scale will diminish for a very long and thin (slender) beam. Also, Fig. 4 illustrates that the nonlocal Timoshenko beam frequency approaches the local Timoshenko beam frequency as the slenderness increases.

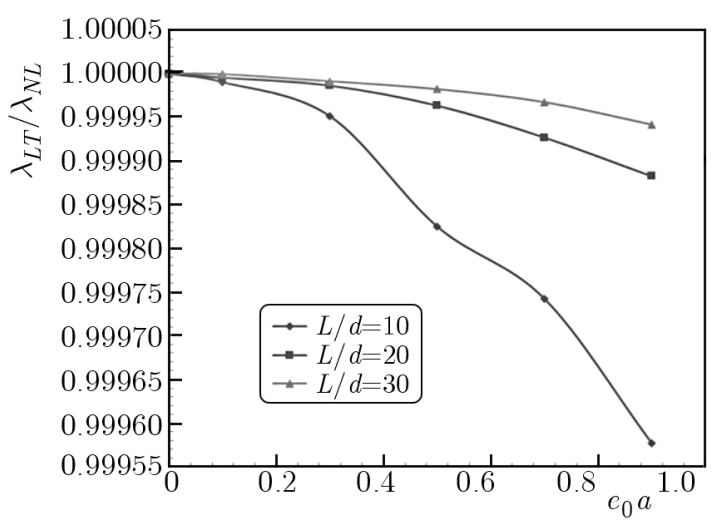

Fig. 3. Small scale effect on the frequency ratio with different values of $L / d$ (both ends clamped) 
Table 6. First three frequencies $\sqrt{\lambda}$ of the nonlocal Timoshenko beam with two kinds of boundary conditions

\begin{tabular}{|c|c|c|c|c|}
\hline$e_{0} a$ & Mode & $L / d=10$ & $L / d=20$ & $L / d=30$ \\
\hline \hline \multicolumn{3}{|c|}{ Simply supported-simply supported beam } \\
\hline 0 & & 3.08057 & 3.12577 & 3.13451 \\
0.1 & & 3.08056 & 3.12577 & 3.13451 \\
0.3 & 1 & 3.08056 & 3.12577 & 3.1345 \\
0.5 & & 3.08052 & 3.12576 & 3.1345 \\
0.7 & & 3.08047 & 3.12574 & 3.13449 \\
0.9 & & 3.08040 & 3.12572 & 3.13448 \\
\hline 0 & & 5.94588 & 6.18907 & 6.24037 \\
0.1 & & 5.94584 & 6.18906 & 6.24036 \\
0.3 & & 5.94558 & 6.18898 & 6.24033 \\
0.5 & 2 & 5.94466 & 6.18882 & 6.24025 \\
0.7 & & 5.94425 & 6.18858 & 6.24015 \\
0.9 & & 5.94318 & 6.18826 & 6.24000 \\
\hline 0 & & 8.53236 & 9.15198 & 9.29787 \\
0.1 & & 8.53225 & 9.15194 & 9.29785 \\
0.3 & 3 & 8.53139 & 9.15165 & 9.29771 \\
0.5 & & 8.52995 & 9.15107 & 9.29743 \\
0.7 & & 8.52936 & 9.15020 & 9.29702 \\
0.9 & & 8.52366 & 9.14904 & 9.29647 \\
\hline \multicolumn{5}{|c|}{ Clamped-simply } \\
\hline 0 & & 3.829744 & 3.901179 & 3.915187 \\
0.1 & & 3.829726 & 3.901175 & 3.915186 \\
0.3 & & 3.829653 & 3.901155 & 3.915176 \\
0.5 & 1 & 3.829491 & 3.901111 & 3.915155 \\
0.7 & & 3.829248 & 3.901045 & 3.915136 \\
0.9 & & 3.828925 & 3.900957 & 3.915086 \\
1 & & 3.828732 & 3.900904 & 3.915063 \\
\hline 0 & & 6.644277 & 6.948166 & 7.01359 \\
0.1 & & 6.644219 & 6.948148 & 7.013581 \\
0.3 & & 6.642754 & 6.948008 & 7.013516 \\
0.5 & 2 & 6.642824 & 6.947726 & 7.013386 \\
0.7 & & 6.641431 & 6.947305 & 7.013191 \\
0.9 & & 6.639576 & 6.946740 & 7.01293 \\
1 & & 6.638475 & 9.946408 & 7.012778 \\
\hline 0 & & 9.177342 & 9.888691 & 10.05988 \\
0.1 & & 9.177189 & 9.888635 & 10.05986 \\
0.3 & & 9.175962 & 9.888215 & 10.05966 \\
0.5 & 3 & 9.173494 & 9.887381 & 10.05925 \\
0.7 & & 9.169836 & 9.886127 & 10.05866 \\
0.9 & & 9.164950 & 9.883445 & 10.05853 \\
1 & & 9.162052 & 9.883461 & 10.05806 \\
\hline
\end{tabular}




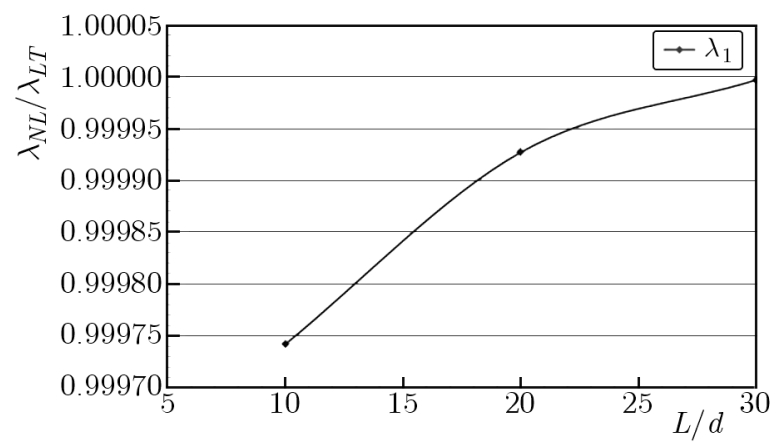

Fig. 4. Effect of slenderness on the nonlocal beam frequency $(\alpha=0.7$, both ends clamped)

\section{Conclusion}

A semi-analytical method called the differential transformation method is generalized to analyze vibration characteristics of a nanobeam. The formulation is based on the assumptions of Timoshenko beam theory and the nonlocal differential constitutive relations of Eringen. The transverse shear force and rotary inertia that become significant at short beams and higher frequencies are taken into account in the equations. Also, the effect of the small scale coefficient as well as the slenderness and boundary conditions in various frequency ratios are investigated. It is demonstrated that the DTM has high precision and computational efficiency in the vibration analysis of nanobeams.

\section{References}

1. Abdel-Halim Hassan I.H., 2002, On solving some eigenvalue problems by using a differential transformation, Applied Mathematics and Computation, 127, 1, 1-22

2. Aifantis E.C., 1984, On the microstructural origin of certain inelastic models, Journal of Engineering Materials and Technology, 106, 4, 326-330

3. Amara K., Tounsi A., Mechab I., Adda-Bedia E.A., 2010, Nonlocal elasticity effect on column buckling of multiwalled carbon nanotubes under temperature field, Applied Mathematical Modeling, 34, 12, 3933-3942

4. Baughman R.H., Zakhidov A.A., De Heer W.A., 2002, Carbon nanotubes - the route toward applications, Science, 297, 5582, 787-792

5. Chen C.O.K., Ju S.P., 2004, Application of differential transformation to transient advectivedispersive transport equation, Applied Mathematics and Computation, 155, 1, 25-38

6. Chow T.L., 2013, Classical Mechanics, CRC Press, Boca Raton, Florida, USA

7. ERINGen A.C., 1972a, Linear theory of nonlocal elasticity and dispersion of plane waves, International Journal of Engineering Science, 10, 5, 425-435

8. ERINGen A.C., 1972b, Nonlocal polar elastic continua, International Journal of Engineering Science, 10, $1,1-16$

9. ERINGEN A.C., 1983, On differential equations of nonlocal elasticity and solutions of screw dislocation and surface waves, Journal of Applied Physics, 54, 9, 4703-4710

10. Eringen A.C., Edelen D.G.B., 1972, On nonlocal elasticity, International Journal of Engineering Science, 10, 3, 233-248

11. Lee Y.Y., Wang C.M., Kitipornchai S., 2003, Vibration of Timoshenko beams with internal hinge, Journal of Engineering Mechanics, 129, 3, 293-301

12. Leissa A.W., QAtu M.S., 2011, Vibration of Continuous Systems, McGraw Hill Professional 
13. Li C., Chou T.W., 2003, A structural mechanics approach for the analysis of carbon nanotubes, International Journal of Solids and Structures, 40, 10, 2487-2499

14. LiEw K.M., Hu Y., HE X.Q., 2008, Flexural wave propagation in single-walled carbon nanotubes, Journal of Computational and Theoretical Nanoscience, 5, 4, 581-586

15. Lu P., Lee H.P., Lu C., Zhang P.Q., 2006, Dynamic properties of flexural beams using a nonlocal elasticity model, Journal of Applied Physics, 99, 7, 073510

16. Maranganti R., Sharma P., 2007, Length scales at which classical elasticity breaks down for various materials, Physical Review Letters, 98, 19, 195504

17. Mindlin R.D., 1964, Micro-structure in linear elasticity, Archive for Rational Mechanics and Analysis, 16, 51-78

18. Peddieson J., Buchanan G.R., McNitt R.P., 2003, Application of nonlocal continuum models to nanotechnology, International Journal of Engineering Science, 41, 3, 305-312

19. Wang C.M., Reddy J.N., Lee K.H., Eds., 2000, Shear Deformable Beams and Plates: Relationships with Classical Solutions, Elsevier

20. Wang C.M., Zhang Y.Y., He X.Q., 2007, Vibration of nonlocal Timoshenko beams, Nanotechnology, 18, 10, 105401

21. WANG Q., 2005, Wave propagation in carbon nanotubes via nonlocal continuum mechanics, Journal of Applied Physics, 98, 12, 124301

22. WANG Q., VARADAN V.K., 2006, Vibration of carbon nanotubes studied using nonlocal continuum mechanics, Smart Materials and Structures, 15, 2, 659

23. WAng X., CAi H., 2006, Effects of initial stress on non-coaxial resonance of multi-wall carbon nanotubes, Acta Materialia, 54, 8, 2067-2074

24. WAng Z.G., 2013, Axial vibration analysis of stepped bar by differential transformation method, Applied Mechanics and Materials, 419, 273-279

25. Xu M., 2006, Free transverse vibrations of nano-to-micron scale beams, Proceedings of the Royal Society A: Mathematical, Physical and Engineering Science, 462, 2074, 2977-2995

26. Zhang Y.Q., Liu G.R., Wang J.S., 2004, Small-scale effects on buckling of multiwalled carbon nanotubes under axial compression, Physical Review B, 70, 20, 205430

27. Zhang Y.Q., Liu G.R., XIE X.Y., 2005, Free transverse vibrations of double-walled carbon nanotubes using a theory of nonlocal elasticity, Physical Review B, 71, 19, 195404

28. Zhou J.K., 1986, Differential Transformation and its Applications for Electrical Circuits, Huazhong University Press, Wuhan, China

29. Zhu H., Wang J., Karihaloo B., 2009, Effects of surface and initial stresses on the bending stiffness of trilayer plates and nanofilms, Journal of Mechanics of Materials and Structures, 4, 3, 589-604 